\title{
Research on Planning Methods of Three-dimensional tactical airspace
}

\author{
Wang jianbo \\ Air Traffic Control and Navigation College, \\ Air Force Engineering University, \\ Xian, China \\ e-mail: jianbowang2008@163.com
}

\author{
Ma Jiacheng \\ Air Traffic Control and Navigation College, \\ Air Force Engineering University, \\ Xian, China \\ e-mail: 727032612@qq.com
}

\begin{abstract}
Three-dimensional tactical training Airspace planning is a complex combinatorial optimization problem. It is very important to construct the corresponding model and design the efficient and fast algorithm to improve the efficiency of airspace utilization and training. Aiming at the characteristics of airspace, this paper divides the whole airspace into cubical units. Based on this, the training airspace planning model is constructed, and an improved genetic algorithm is used to find the optimal solution, which excludes a large number of infeasible solutions and improves the convergence rate. The experimental results show that the method is feasible and stable, and has a strong practical value, which can effectively solve the planning problem of $3 \mathrm{D}$ tactical training airspace.
\end{abstract}

Keywords-component; formatting; style; styling;

\section{INTRODUCTION}

With the advance of Chinese air force military training transformation, military training time, the proportion of tactical training continues to increase. Multi-military joint training and system confrontation has become the norm. At the same time, Chinese civil air transport industry steps into a new stage of sustained and rapid development. Thus, the demand for airspace is also sharply increased. Therefore, rational planning and use of air tactical training airspace is conducive to take advantage of the army main battle equipment and the rational optimization of airspace structure. These can effectively alleviate the contradiction between military and civil aviation airspace requirements.

Three-dimensional tactical training airspace planning is a centralized planning for the airspace required for the air force tactical training. It means to arrange a series of different size of the airspace block in the jurisdiction of the airspace according to the different requirements of training subjects. The different blocks can not overlap. Three-dimensional tactical training airspace planning is to seek the airspace planning program maximized the utilization of airspace .Theoretically, this problem is similar to the three-dimensional packing problem, which is a typical NP-Hard problem [1]. At present, the research on twodimensional packing problem is more [2 4], three-dimensional packing problem has less research because of complexity and difficulty[5]. Because of its unique airspace, the planning problem is different from the packing problem. Due to the need to consider the dimension, shape and other factors, the computational complexity is extremely high. At present, the training of airspace planning is mainly based on the experience of staff officers, resulting in lower utilization of airspace.

In the process of solving such problems, there are the following difficulties: the use of exhaustive method can not accurately find the best solution because there are "combinatorial explosion" in the process. For the heuristic search method, the heuristic information mainly rely on human experience. There is also a "combinatorial explosion". For the neural network method, it can easily fall into local optimum, although the effect is obvious, especially it has a wide range of the airspace block. It is different to find a global optimal solution. In this paper, the airspace is divided into cubes, and the airspace discretization model is constructed. The improved genetic algorithm is used to find the optimal plan and obtain good experimental results.

\section{Training Airspace Planning Mathematical Model}

Tactical training Airspace planning is to arrange the various training subjects within the jurisdiction of the airfield's airspace. The rational planning of the training airspace is helpful for the the utilization of the entire airspace. In this paper, the whole airspace and the airspace required for each training subject are abstracted as a collection of unit cubes. Due to the influence of the regional topography, air no-fly zone, military and civilian routes, the training airspace is usually irregular shape. The discretization of the airspace is necessary to seek the optimal airspace planning program. For the training airspace planning problem, it can be solved in the three-dimensional Cartesian coordinate system. The constraints are as follows:

1) Space constraints: Each training airspace block should be arranged in the airspace under the jurisdiction of the airport, and the total volume of airspace required is not greater than the total airspace volume of the airport.

2) Position constraints: the training space between the blocks can not overlap.

3) Directional constraints: Considering the actual situation, the training space block placement need to parallel with the axis, but it can be rotated 90 degrees horizontally. 
Symbol Description

The coordinate system used in this paper is 3D Cartesian Cartesian coordinate system. The discretized spatial domain is placed in the three-dimensional coordinate system and its side is parallel with the horizontal axis of the coordinate axis. The left and the bottom of the vertex is as a reference point. The specific parameters and variables required in this paper are described as follows:

$\mathrm{n}$ : the total number of training airspace block;

$\mathrm{m}$ : the number of training airspace blocks that are finally exhausted;

$\mathrm{x}_{\mathrm{i}}, \mathrm{y}_{\mathrm{i}}, \mathrm{z}_{\mathrm{i}}$ : the reference point coordinates of training space block, $\mathrm{i}=1,2 \ldots \ldots \mathrm{n}$;

$l_{i}, w_{i}, h_{i}$ : Length, width, height of the training space block (lengrh is greater than width), $i=1,2 \ldots \ldots n$;

$\mathrm{V}_{\mathrm{i}}$ : volume of training airspace block, $\mathrm{V}_{\mathrm{i}}=\mathrm{l}_{\mathrm{i}} * \mathrm{w}_{\mathrm{i}} * \mathrm{~h}_{\mathrm{i}}, \mathrm{i}=1,2 \ldots \mathrm{n}$;

$\mathrm{V}$ : the volume of the entire airspace after discretization;

$a_{i}$ : a variable of value 0 or $1, a_{i}=1$ if the training airspace block is arranged in the airspace, $a_{i}=1, i=1,2 \ldots n$;

$E_{x y z}$ : A variable of value 0 or 1 indicating whether the spatial point $(x, y, z)$ is occupied. $E_{x y z}=1$ if occupied, otherwise $\mathrm{E}_{\mathrm{xyz}}=0$;

$\mathrm{L}_{\mathrm{xi}}, \mathrm{l}_{\mathrm{yi}}, \mathrm{l}_{\mathrm{zi}}$ : A variable of value 0 or 1 indicates whether the length of the training space block $\mathrm{i}$ is parallel to an axis of the $\mathrm{X}$-axis, Y-axis or Z-axis. If parallel to the $\mathrm{X}$-axis, $\mathrm{l}_{\mathrm{xi}}=1, \mathrm{i}=1,2 \ldots \mathrm{n}$;

$\mathrm{W}_{\mathrm{xi}}, \mathrm{W}_{\mathrm{yi}}, \mathrm{W}_{\mathrm{zi}}$ : A variable of value 0 or 1 indicates whether the width of the training space block $\mathrm{i}$ is parallel to one axis of the $\mathrm{X}$ axis, $\mathrm{Y}$ axis or $\mathrm{Z}$ axis. If parallel to the $\mathrm{Y}$ axis, $\mathrm{W}_{\mathrm{xi}}=1, \mathrm{i}=1,2 \ldots \mathrm{n}$;

$\mathrm{h}_{\mathrm{xi}}, \mathrm{h}_{\mathrm{yi}}, \mathrm{h}_{\mathrm{zi}}$ : A variable of value 0 or 1 indicates whether the height of the training block $\mathrm{i}$ is parallel to the axis of $\mathrm{X}, \mathrm{Y}$ or $\mathrm{Z}$.

If it is parallel to $\mathrm{z}, \mathrm{h}_{\mathrm{xi}}=1, \mathrm{i}=1,2 \ldots \mathrm{n}$;

Objective Function and Constraints

In this paper, the optimization goal is to maximize the utilization of the entire airspace, that is, in the airspace under the jurisdiction of the airport to arrange the training of airspace block up, so the objective function can be expressed as:

$$
\operatorname{Max} \mathrm{Z}=\sum_{i=1}^{n}\left(v_{i} \bullet a_{i}\right)
$$

Constraints can be expressed as:

$$
E_{x y z}+B_{i}^{x y z} \leq 1, i \in\{1,2, \cdots, n\}
$$

$\frac{B_{i}^{x y z}+B_{j}^{x y z} \leq 1, i, j \in\{1,2, \cdots, n\} a n d i \neq j}{\left\{\begin{array}{l}l_{x i}+l_{y i}+l_{z i}=1 \\ w_{x i}+w_{y i}+w_{z i}=1 \\ h_{x i}+h_{y i}+h_{z i}=1 \\ l_{x i}+w_{x i}+h_{x i}=1 \\ l_{y i}+w_{y i}+h_{y i}=1 \\ l_{z i}+w_{z i}+h_{z i}=1\end{array} \quad i \in\{1,2, \cdots, n\}\right.}$

Condition (2) is to ensure that the airspace blocks do not overlap each other; condition (3) is representative of the direction of the training space block constraints (1) (4) is to ensure that all training airspace blocks are placed within the jurisdiction of the training airspace;

\section{Genetic Algorithm for Airspace Planning}

Genetic algorithm is mainly to find the best solution to the problem through the processing parameters of the code set and the use of evaluation function without other prerequisites, which also brought great convenience to the solution of the problem . According to the characteristics of training airspace planning, this paper discretizes the airspace, compiles the corresponding encoding and decoding methods, and solves the constraint problem by punishing technology. Compared with the artificial division, the assistant of the computers greatly shorten the working hours. To a certain extent, it can ensure the utilization of airspace.

Training Airspace Pretreatment

For training areas administered by military airports, these are usually irregular due to the terrain and the effects on the route. In order to make a more reasonable use of airspace, we must firstly discretize the boundary of airspace. Specific steps are as follows:

Step1. The entire training airspace stored in memory in the form of images.

Step2. Convert the image to a bitmap ( $0 / 1$ form) for storage. 
Step3. Artificial division of the interval, and take the reliability of 0.75 , the bitmap change into a matrix V.

Through the above operation, each training airspace is stored in the form of a matrix for subsequent operations.

\section{Encoding and Decoding}

Coding is to convert the feasible solution of the problem to the search space that can be handled in the genetic algorithms. In this paper, the encoding is mainly to consider the placement of airspace direction. Taking into account the actual situation, in this paper, the horizontal block only make the 90 degrees of rotation. Each scheme corresponds to a string $S=\left\{S_{1}, S_{2}, \ldots, S_{n}\right.$, $\left.S_{n+1}, \ldots S_{2 n}\right\}$ with an encoding length of $2 n$ ( $n$ is the total number of spatial blocks). $S_{n+1} \sim S_{2 n}$ is the direction of placement of the corresponding airspace block, which is composed of $\{0,1\}$ characters. A string of 0 means that the length is parallel to the $\mathrm{x}$-axis, and 1 means that the length is flush with the $\mathrm{y}$-axis, that is, 90 degrees horizontally.

Decoding is to get the fitness function value according to the special rules for further processing. In this paper, the principle is to find the corresponding space in the airspace according to the order of the string and the corresponding placement direction. For the placement of airspace block, the use of layer by layer method can be used for each layer. For each layer, the lower left priority strategy can be adopted .

\section{Evaluation Functions}

The main goal of airspace planning is to improve the airspace utilization, so the objective function is as a fitness function (S represents a chromosome). which is:

$$
\text { Fitness }(s)=\left\{\begin{array}{l}
\sum_{i=1}^{n}\left(v_{i}^{\bullet} a_{i}\right) / V+m / n \quad \text { if } \sum_{i=1}^{n}\left(v_{i} \bullet a_{i}\right)<v \\
0 \quad \text { else }
\end{array}\right.
$$

However, due to the constraints on the chromosome, the penalty function is used to transform the constrained problem into unconstrained problem. For the airspace planning problem, this paper mainly consider the following three penalty items:

1) The space block must be placed within the jurisdiction of the airspace, that is, to meet the constraints (1) (4);

$$
\mathrm{q}_{1}(S)=\left\{\begin{array}{l}
1, \text { if } \boldsymbol{E}_{x y z}+B_{i}^{x y z} \leq 1 \text { and } \sum_{i=1}^{n}\left(V_{i} \bullet a_{i}\right) \leq V \\
0, \text { else }
\end{array}\right.
$$

The space blocks can not overlap, that is to meet the constraints (2);

$$
\mathbf{q}_{2}(S)=\left\{\begin{array}{l}
1, \text { if }+B_{j}^{x y z} \leq 1 \\
0, \text { else }
\end{array}\right.
$$

2) The spatial block needs to be placed parallel to the coordinate axis, that is, the constraint (3) is satisfied;

$$
\mathbf{q}_{3}(S)=\left\{\begin{array}{l}
1, \text { true } \\
0, \text { false }
\end{array}\right.
$$

For any of the three constraints, the solution is infeasible. Therefore, the evaluation function is defined as:

$$
\operatorname{Eval}(S)=\operatorname{Fitness}(s) \cdot\left(\sum_{\mathrm{k}=1}^{3} \boldsymbol{q}_{k}(S)\right)
$$

\section{Genetic Operation Process}

For a certain chromosome, the genetic manipulation carried out in this paper includes: selection operation, crossover operation and mutation operation. the evolution of species in nature is simulated through these operations to constantly seek the optimal solution to the problem.

\section{Select Operation}

The purpose of the selection operation is to select the best individual from the current population as the parent of the next generation, which is selected by the fitness function to be inherited into the next generation. In this paper, a combination of roulette and the best individual preservation method are used to select the individuals. The idea of roulette selection is to firstly calculate the fitness function of each individual and sum. Then according to the proportion of each individual, the next generation individuals are selected. In order to ensure the optimal evolution of the population, this paper also uses the best individual preservation method. That is: the father of the highest fitness value of the individual inherited to the next generation. This ensures that the genetic algorithm converges with a probability of 1.

\section{Cross Operation}

Crossover refers to the intercrossing of two individuals in a way to exchange part of the gene to form two new individuals. In this paper, we use sequential and two-point crossover. During the intersection, two integers a1 and a2 (a1 <a2)(between $[1,2 \mathrm{n}])$ are randomly generated as cross points. If a1 $>\mathrm{n}$, then the gene between a1 $\sim$ a2 use the method of order crossing [6]; if a1 $>\mathrm{n}$, then two-point cross method is adopted between the a1 $\sim$ a2 genes, namely: direct exchange cross the selected genes, other genes remain unchanged. If $\mathrm{a} 1 \leqslant \mathrm{n}$ and $\mathrm{a} 2>\mathrm{n}$, then the order cross is adopted between the $\mathrm{a} 1 \sim$ an genes, and the twopoint cross method is adopted between the an $+1 \sim$ a2 genes. 


\section{Mutation Operation}

In order to ensure the diversity of the population during the genetic process and avoid the premature convergence of the algorithm that it can not find the global optimal individuals, the mutation operation is carried on the individuals with certain probability. In this paper, the mutation operation is only for the latter part of the gene. The random number is generated for every gene between $[n+1,2 n]$. If it is smaller than the mutation rate, the genes were replaced with other alleles.

Simulation Analysis

In order to test the effectiveness of this method for training airspace planning problems, the following example simulations are performed.

(1) The airspace size required for different tactical training courses is shown in Table 1 below:

\begin{tabular}{cccccccc} 
No & Length & Width & Height & No & Length & Width & Height \\
\hline 1 & 8 & 3 & 4 & 2 & 5 & 5 & 3 \\
3 & 12 & 4 & 2 & 4 & 16 & 8 & 2 \\
5 & 10 & 10 & 1 & 6 & 14 & 7 & 2 \\
7 & 14 & 14 & 3 & 8 & 10 & 5 & 3 \\
9 & 10 & 11 & 4 & 10 & 12 & 10 & 2 \\
11 & 7 & 7 & 2 & & & &
\end{tabular}

The training space under the jurisdiction of the airport as shown in Figure 1:

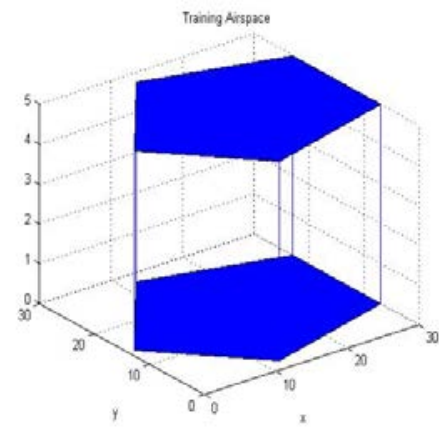

Fig.1 Training Airspace



Fig.2 Discrete Training Airspace

The irregular training airspace is discretized and transformed into the corresponding matrix. The discretization of the airspace is shown in Figure 2 above.

The initial genetic parameters were calculated as follows: population size $m=20$, iteration algebra $n=100$, initial crossover rate $\mathrm{cp}=0.9$, and initial mutation rate $\mathrm{mp}=0.1$. The calculated maximum fitness value is 1.6555 . The fitness value is shown in Figure 3 below. 


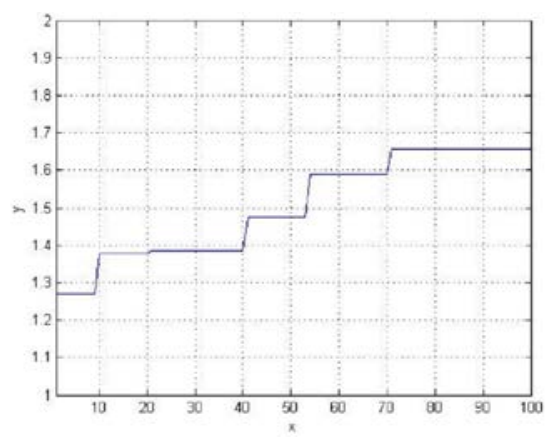

Fig.3 Variation curve of fitness value

Finally, the tactical training airspace planning and bottom of the distribution chart are shown in Figure 4 and Figure 5:

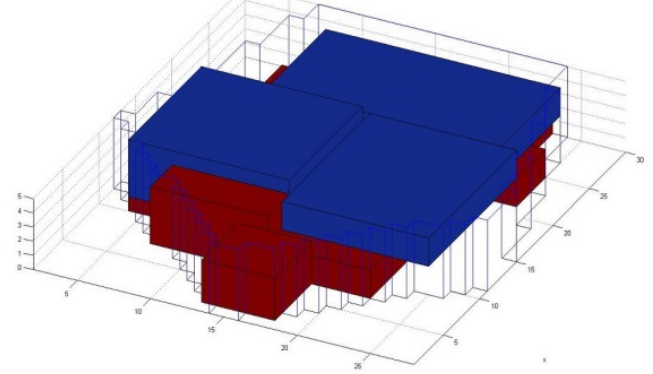

Figure 4 training airspace planning diagram

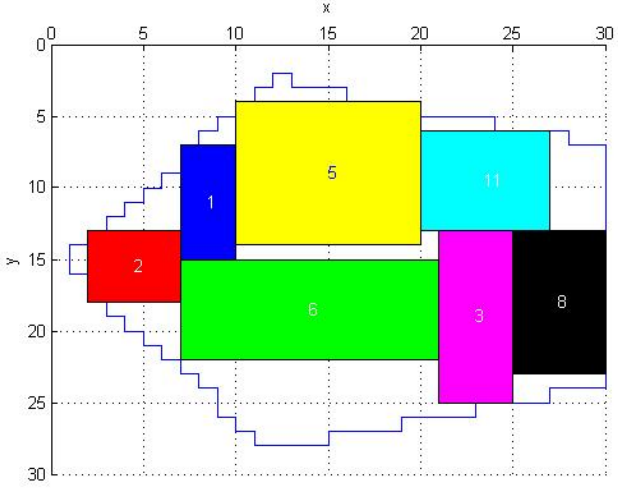

Figure 5 Bottom surface distribution

(2) It is assumed that there are only three types of training subjects in a certain period of time. The required airspace size is $(4,14,3)(8,8,2)(10,5,3)$. Using the above method, the optimal programming can also be obtained. The optimal fitness value is 1.7040. Figure 6 and Figure 7 show the tactical training airspace planning and fitness values.

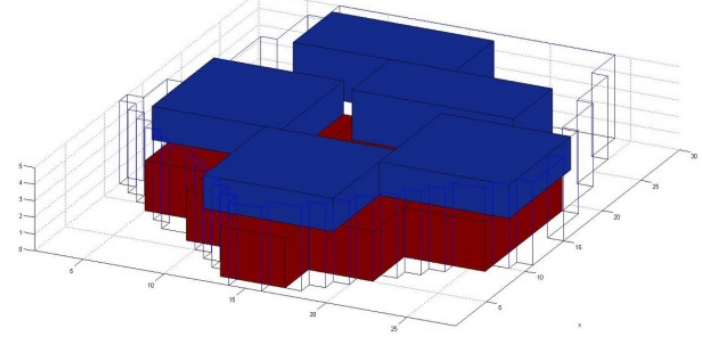

Figure 6 training airspace planning diagram 


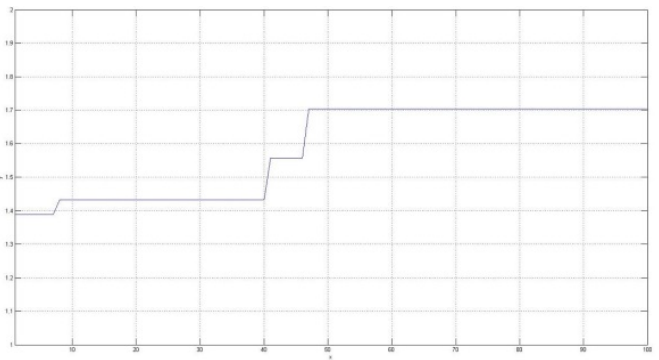

Figure.7 Curve of fitness value

From the above example, this method has strong applicability for training airspace planning. This method can quickly find the optimal planning scheme. Compared with the artificial division, the assistant of computer greatly shorten the nesting time and improve the utilization of airspace. The comparison between the two examples shows that when the number of training subjects is large, the optimization effect of this method will be more obvious.

\section{CONCLUSION}

With the increasing number of aviation tactical training courses, dividing the airspace by the staff offices according to the experience can not guarantee the efficient use of airspace. In this paper, three-dimensional airspace is discretized, and the corresponding planning model is built according to the characteristics of spatial planning. The improved genetic algorithm is used to find the optimal planning scheme, and the feasibility and effectiveness of the method are verified by an example. Compared with the artificial division of training area, using this method can improve the utilization of airspace. But the stability and efficiency of the algorithm need to be further studied and improved.

\section{ACKNOWLEDGMENT}

In this paper, the research was sponsored by the Key Technique of Unmanned Aerial Vehicle Airspace Safety (KGKT05140501)

\section{REFERENCES}

[1] PisingerD. Heuristics for the container loading problem[J].European Journal of Operational Research,2002,141(2): 382-392

[2] Khoo W S, Saratchandran P, Sundararajan N. A genetic approach for two dimensional parking with constraints[A].Proceedings of the Part II International Conference on Computational Science ICCS[C].San Francisco,CA, USA: Alexandrov, 2001. 291-299

[3] MiyazawaF K, Wakahayashi Y. Packing problems with orthogonal rotations[ A].Proceedings of the 6th Latin American Symposiumon Theoretical Informatics[C].BuenosAires, Argentina: Farach-ColtonM,2004. 359-368.

[4] Imahori S, Yagiura M, Iharaki T. Local search algorithmsfor the rectangle packing problem with general spatial costs[J]. Math Program,2003,B97: 543-569

[5] LIU Jia-min,Ma Guang-kun,HUANG You-qun.Rearch of a Heuristic Algorithm for 3D Container Loading Based on Comnination[J].Journal of Engineering Graphics,2005(1):22-25)

[6] WANG Xiaoping,CAO Liming.Theory,Application and Software Realization of GA[M].xi’an:Publishing of xi’an Traffic University,2002.

[7] Jiang Jjinshan,Lin Zhengchun.Solving 2D Bin-packing Problem on Self-adaptive Genetic Algorithm[J].Computer Application and Solfware,2008,25(7):244-246

[8] Martello Silvano, Pisinger, David, and Vigo,Daniele.The Three-Dimensional Bin Packing Problem(J). Operations Research, 2000 Informs. Vol.48: 256-267. 